\title{
A high-fidelity heralded quantum squeezing gate
}

\author{
Jie Zhao, ${ }^{1}$ Kui Liu, ${ }^{2}$ Hao Jeng, ${ }^{1}$ Mile Gu ${ }^{3,4}$ Jayne Thompson, ${ }^{4}$ Ping Koy Lam ${ }^{1 *}$ and Syed M Assad, ${ }^{1}$ \\ ${ }^{1}$ Centre of excellence for Quantum Computation and Communication Technology, \\ Department of Quantum Science, Research School of Physics and Engineering, \\ The Australian National University, Canberra ACT 2601, Australia. \\ ${ }^{2}$ State key laboratory of quantum optics and quantum optics devices, \\ Institute of Opto-Electronics, Collaborative Innovation Center of Extreme Optics, \\ Shanxi University, Taiyuan 030006, China. \\ ${ }^{3}$ School of Physical and Mathematical Sciences, Nanyang \\ Technological University, 50 Nanyang Ave, 639798, Singapore. \\ ${ }^{3}$ Complexity Institute, Nanyang Technological University, 50 Nanyang Ave, 639798, Singapore. \\ ${ }^{4}$ Centre for Quantum Technologies, National University of \\ Singapore, Block S15, 3 Science Drive 2, 117543, Singapore.
}

(Dated: June 2, 2020)

\section{INTRODUCTORY PARAGRAPH}

A universal squeezing gate capable of squeezing arbitrary input states is essential for continuous-variable quantum computation $[1,2]$. However, in present stateof-the-art techniques [3, 4], the fidelity of such gates is ultimately limited by the need to create squeezed vacuum modes of unbounded energy. Here we circumvent this fundamental limitation by using a heralded squeezing gate. We propose and experimentally demonstrate a squeezing gate that can achieve near unit fidelity for coherent input states. In particular, for a target squeezing of $2.3 \mathrm{~dB}$, we report a fidelity of $98.5 \%$. This result cannot be reproduced by conventional schemes even if the currently best available squeezing of $15 \mathrm{~dB}[5]$ is utilised when benchmarked on identical detection inefficiencies. Our technique can be applied to non-Gaussian states and provides a promising pathway towards high-fidelity gate operations and fault-tolerant quantum computation.

\section{MAIN SECTION}

Gaussian operations are essential building blocks for continuous-variable quantum information processing. With the exception of the squeezing operation, all other Gaussian operations can be realised readily with near unit fidelity in quantum optics. However, current capability to implement squeezing operations with high fidelity is still limited. This is unfortunate since the squeezing operation is a prerequisite for universal quantum computation $[1,2]$. It is also a necessary component for many other fundamental quantum operations like the controlled- $Z$ gate [6-8], the quantum non-demolition gate [9], the control phase gate [10] and quantum error correction [11]. Moreover, its application to nonGaussian states facilitates quantum information tasks such as decoherence mitigation [12], preparation of nonclassical states [13] and quantum state discrimination of coherent-state qubits [14].

While extensive effort has been devoted to the gener- ation of squeezed vacuum [5, 15], the development of a universal squeezing gate that can act on arbitrary input states has been lagging behind. Recent experiments have successfully generated a squeezed vacuum with a squeezing magnitude of $15 \mathrm{~dB}$ [5]. In contrast, demonstrations of a universal squeezing gate have only attained $1.2 \mathrm{~dB}$ for a reliable fidelity of $94 \%[16,17]$. High squeezing levels for vacuum inputs is possible through the parametric amplification process in an optical cavity. However, extending this method to arbitrary input states presents significant challenges due to cavity loss $[13,18,19]$ and interference effects [20, 21].

Instead, current state-of-the-art implementations of a universal squeezing gate use an ancillary squeezed vacuum as a resource to drive the squeezing gate $[3,4,16$, 17]. Once the ancillary state has been prepared, the squeezing gate can be implemented using Gaussian measurements and feed-forward operations. However, highly squeezed ancilla are required to achieve a reasonable fidelity. Unit fidelity can only be achieved with an infinitely squeezed ancilla. Thus in realistic implementations, the output fidelity will always be limited.

Here, we present and experimentally demonstrate a heralded squeezing gate that overcomes this limitation. A heralding filter is implemented in the feed-forward operation whereby an enhancement in fidelity can be achieved by increasing the filter strength without requiring more squeezing resources. In contrast to conventional implementations, this scheme can approach unit fidelity. With the inclusion of the present squeezing gate, we thus have a complete set of Gaussian operations that can be implemented with high fidelity.

The universal squeezing gate performs the unitary operation $S\left(r_{\mathrm{t}}\right)=\exp \left[\frac{1}{2}\left(r_{\mathrm{t}}^{*} a^{2}-r_{\mathrm{t}} a^{\dagger 2}\right)\right]$, where $a$ is the annihilation operator and $r_{\mathrm{t}}$ is the target squeezing strength. Our squeezing gate is illustrated in Fig. 1. First, an optical parametric amplifier is used to produce an ancillary squeezed vacuum with squeezing parameter $r_{\mathrm{a}}$. Next, an input state $\rho_{\text {in }}$ is mixed with the ancillary state on a beam splitter with transmissivity $t_{\mathrm{s}}$. The reflected mode is then split on a beam splitter with trans- 
missivity $t_{\mathrm{m}}$ so that its amplitude and phase quadratures can be simultaneously measured. The measurement outcome, denoted by a complex number $\alpha_{\mathrm{m}}$, is used to herald a successful squeezing operation by employing a probabilistic filter. The gate is successfully heralded with probability (see Methods)

$$
P_{\mathrm{f}}\left(\alpha_{\mathrm{m}}\right)= \begin{cases}\exp \left[\left(1-\frac{1}{g_{\mathrm{f}}}\right)\left(\left|\alpha_{\mathrm{m}}\right|^{2}-\alpha_{\mathrm{c}}^{2}\right)\right] & \text { for }\left|\alpha_{\mathrm{m}}\right|<\alpha_{\mathrm{c}}, \\ 1 & \text { for }\left|\alpha_{\mathrm{m}}\right| \geq \alpha_{\mathrm{c}} .\end{cases}
$$

This filter function, which was previously used to emulate a noiseless linear amplifier [22-24], depends on two parameters: the filter strength $g_{\mathrm{f}} \geq 1$, and the cutoff parameter $\alpha_{\mathrm{c}}$. A large filter strength will result in a higher output fidelity at the expense of a lower success probability. When $g_{\mathrm{f}}=1$, the heralded squeezing gate reduces to the conventional squeezing gate. The cut-off parameter determines the operational regime; a larger cut-off will allow for the squeezing of states with a higher mean photon numbers. Finally, upon a successful heralding event, the measurement outcomes of amplitude and phase are rescaled by the electronic gains $g_{\mathrm{x}}$ and $g_{\mathrm{y}}$, and fed-forward to the transmitted mode to complete the squeezing operation. The target squeezing level is determined by the ancillary squeezing level and the transmissivity of the two beam splitters (see Supplementary section I).

The faithfulness of a squeezing gate is typically benchmarked by the fidelity between the output and ideal target state. For Gaussian inputs within the operational regime, this fidelity is independent of the input quadrature amplitudes. This is because we operate at the unitygain point where the mean quadrature amplitudes of the output and target coincide (see Supplementary section I).

Figure 2 illustrates the trade-off between fidelity, target squeezing, and success probability. We identify two operational regimes distinguished by the filter strength. When the filter strength is low, we operate in the first regime which exhibits a favourable success probability. The majority of fidelity enhancement can be obtained without dropping below $1 \%$ success probability. Regardless of the target squeezing, a significant improvement in fidelity is obtained compared to the conventional approach. In the second regime, a high filter strength allows near unit fidelity for any target squeezing $r_{\mathrm{t}} \leq r_{\mathrm{a}}$, which is impossible conventionally. An attractive feature of our scheme is that we can choose to operate in either regime by simply tuning the filter strength without reconfiguring the experimental setup.

We now report the experimental results. An auxiliary squeezed vacuum with $6.0 \mathrm{~dB}$ squeezing and $6.5 \mathrm{~dB}$ antisqueezing was used as a resource to drive the squeezing gate. In the experiments, we perform a single quadrature measurement by setting $t_{\mathrm{m}}=1$. This allows for a higher success probability compared to a dual quadrature measurement while maintaining comparable fidelity enhancement. In this case, the transmissivity $t_{\mathrm{s}}$ is set according to $t_{\mathrm{s}}=e^{-2 r_{\mathrm{t}}}$ (see Supplementary section I). To test the squeezing gate, we prepared several coherent input states and characterised their outputs by performing homodyne measurements on the amplitude and phase quadratures. We implemented at least $10^{6}$ runs for each input state to generate enough statistics.

Firstly, we present the results for five input states having different phases and with magnitudes $\left|\alpha_{\text {in }}\right|$ ranging from 0.70 to 1.92 in Fig. 3. The target squeezing for these states vary between $2.3 \mathrm{~dB}$ to $10.16 \mathrm{~dB}$. A true squeezing gate operates on arbitrary inputs irrespective of their amplitude or phase. This is verified by the measured outputs of our squeezing gate.

Secondly, we characterise the fidelity as a function of target squeezing in Fig. 4a. The best conventional output fidelity attainable in an idealised experiment using the same ancillary resource but assuming no loss is plotted as a benchmark. We show that this benchmark can be surpassed by increasing the filter strength without requiring a more squeezed ancilla. The trade-off between fidelity and success probability is illustrated in Fig. 4b. For most runs, the success probabilities are greater than $10^{-4}$.

Thirdly, Fig. 5a illustrates the relationship between fidelity and filter strength. The continuous increase in fidelity as a function of filter strength agrees with the theoretical model accounting for experimental imperfections (see Supplementary section IV and Supplementary Fig. 7 for a detailed analysis). The deterministic limit is plotted to identify the minimum filter strength required to exceed this benchmark. We clearly surpass this benchmark for all the data sets.

Finally, Fig. 5b showcases performance of the heralded squeezing gate in the high-fidelity regime when the filter strength is increased to 12.63. For an input magnitude of $\left|\alpha_{\text {in }}\right|=2.91$ and target squeezing of $2.3 \mathrm{~dB}$, we measured a fidelity of $0.985 \pm 0.001$. This fidelity cannot be achieved with the current best squeezed resource [5] in the conventional scheme subject to the same homodyne detection efficiency. Assuming idealised experimental process with zero loss, obtaining this fidelity would require a pure $10.5 \mathrm{~dB}$ squeezed ancilla.

In conclusion, we propose and experimentally demonstrate a heralded squeezing gate that achieves near unit fidelity for coherent inputs while requiring only modest ancillary squeezing. Crucially, heralding circumvents the requirement for a highly squeezed ancilla necessary in conventional methods. The trade-off between fidelity and success probability can be tuned at will, and the majority of fidelity improvement can be achieved without success probability dropping below $1 \%$. This methodology enables us to synthesise squeezing gates to a fidelity that would otherwise be impossible for conventional schemes even with a pure and infinitely squeezed ancillary resource subject to the same experimental loss. In doing so, our techniques complete the set of all Gaussian operations that can be experimentally performed with high 
fidelity.

There are a number of situations where trading determinism for high fidelity squeezing can be useful. Notably, in the Supplementary section II, we illustrate that our techniques can be adapted to squeeze nonGaussian states, such as the single-photon state and the Schrödinger's cat state, with near unit fidelity. This provides a feasible pathway to creating exotic non-classical states [25] which are key resources for computation and engineering sophisticated nonlinear evolutions [26, 27]. High amplitude cat states, for example, are critical to certain models of universal continuous-variable computation [28, 29]. In the cluster state setting, such non-classical states constitute a resource that enables a continuous-variable cluster to perform computations that cannot be efficiently simulated classically [1, 2, 30]. Here, such resources only need to be prepared offline, and a non-deterministic mechanism for synthesising them merely adds an overhead to the preparation procedure. Furthermore, in quantum sensing [28, 29] and illumination, the most physically pertinent resource cost is often the number of photons sent. For example, each probe risks damaging the sample in biological sensing [31], while in covert sensing, each probe risks detection by an adversary [32]. In such scenarios, it becomes quite reasonable to pay a heavier cost during state preparation to maximise the efficacy of each probe. Each of these possibilities merits investigation, whereby one can ascertain the extent to which it is worthwhile to trade determinism for high fidelity.
[1] Mile, G., Weedbrook, C., Menicucci, N. C., Ralph, T. C. \& van Loock, P. Quantum computing with continuousvariable clusters. Phys. Rev. A 79, 062318 (2009).

[2] Menicucci, N. C. Fault-tolerant measurement-based quantum computing with continuous-variable cluster states. Phys. Rev. Lett. 112, 120504 (2014).

[3] Miyata, K. et al. Experimental realization of a dynamic squeezing gate. Phys. Rev. A 90, 060302 (2014).

[4] Ukai, R. et al. Demonstration of unconditional one-way quantum computations for continuous variables. Phys. Rev. Lett. 106, 240504 (2011).

[5] Vahlbruch, H., Mehmet, M., Danzmann, K. \& Schnabel, R. Detection of $15 \mathrm{~dB}$ squeezed states of light and their application for the absolute calibration of photoelectric quantum efficiency. Phys. Rev. Lett. 117, 110801 (2016).

[6] Menicucci, N. C., Ma, X. \& Ralph, T. C. Arbitrarily large continuous-variable cluster states from a single quantum nondemolition gate. Phys. Rev. Lett. 104, 250503 (2010).

[7] Braustein, S. L. Squeezing as an irreducible resource. Phys. Rev. A 71, 055801 (2005).

[8] Yurke, B. Optical back-action-evading amplifiers. J. Opt. Soc. Am. B 2, 732-738 (1985).

[9] Yoshikawa, J.-I. et al. Demonstration of a quantum nondemolition sum gate. Phys. Rev. Lett. 101, 250501 (2008).

[10] Puri, S. \& Blais, A. High-fidelity resonator-induced phase gate with single-mode squeezing. Phys. Rev. Lett. 116, 180501 (2016).

[11] Braustein, S. L. Error correction for continuous quantum variables. Phys. Rev. Lett. 80, 4084-4087 (1998).

[12] Le Jeannic, H., Cavailles A., Huang, K., Filip, R. \& Laurat, J. Slowing quantum decoherence by squeezing in phase space. Phys. Rev. Lett. 120, 073603 (2018).

[13] Miwa, Y. et al. Exploring a new regime for processing optical qubits: Squeezing and unsqueezing single photons. Phys. Rev. Lett. 113, 013601 (2014).

[14] Takeoka, M. \& Sasaki, M. Discrimination of the binary coherent signal: Gaussian-operation limit and simple nongaussian near-optimal receivers. Phys. Rev. A 78, 022320 (2008).

[15] Andersen, U. L., Gehring, T., Marquardt, C. \& Leuchs, G. 30 years of squeezed light generation. Phys. Scr. 91, 053001 (2016).
[16] Yoshikawa, J.-I. et al. Demonstration of deterministic and high fidelity squeezing of quantum information. Phys. Rev. A 76, 060301 (2007).

[17] $\mathrm{Su}, \mathrm{X}$. et al. Gate sequence for continuous variable one-way quantum computation. Nat. Commun. 4, 2828 (2013).

[18] de Oliveira, F. A. M. \& Knight, P. L. Bright squeezing. Phys. Rev. Lett. 61, 830-833 (1988).

[19] La Porta, A., Slusher, R. E. \& Yurke, B. Back-action evading measurements of an optical field using parametric down conversion. Phys. Rev. Lett. 62, 28-31 (1989).

[20] Zhang, J., Ye, C., Gao, F. \& Xiao, M. Phase-sensitive manipulations of a squeezed vacuum field in an optical parametric amplifier inside an optical cavity. Phys. Rev. Lett. 101, 233602 (2008).

[21] Ma, H., Ye, C., Wei, D. \& Zhang, J. Coherence phenomena in the phase-sensitive optical parametric amplification inside a cavity. Phys. Rev. Lett. 95, 233601 (2005).

[22] Fiurášek, J. \& Cerf, N. J. Gaussian postselection and virtual noiseless amplification in continuous-variable quantum key distribution. Phys. Rev. A 86, 060302 (2012).

[23] Chrzanowski, H. M. et al. Measurement-based noiseless linear amplification for quantum communication. Nat. Photon. 8, 333-338 (2014).

[24] Zhao, J., Hao, J. Y., Symul, T., Lam, P. K. \& Assad, S. M. Characterization of a measurement-based noiseless linear amplifier and its applications. Phys. Rev. A 96, 012319 (2017).

[25] Laurat, J., Coudreau, T., Treps, N., Matre, A. \& Fabre, C. Conditional preparation of a quantum state in the continuous variable regime: Generation of a sub-poissonian state from twin beams. Phys. Rev. Lett. 91, 213601 (2003).

[26] Andersen, U. L., Neergaard-Nielsen, J. S., van Loock, P. \& Furusawa, A. Hybrid discrete- and continuous-variable quantum information. Nat. Phys. 11, 713-719 (2015).

[27] van Loock, P. Optical hybrid approaches to quantum information. Laser Photon. Rev. 5, 167-200 (2011).

[28] Ralph, T. C. Coherent superposition states as quantum rulers. Phys. Rev. A 65, 042313 (2002).

[29] Munro, W. J., Nemoto, K., Milburn, G. J. \& Braustein, S. L. Weak-force detection with superposed coherent states. Phys. Rev. A 66, 023819 (2002). 
[30] Menicucci, N. C. et al. Universal quantum computation with continuous-variable cluster states. Phys. Rev. Lett. 97, 110501 (2006).

[31] Taylor, M. A. et al. Biological measurement beyond the quantum limit. Nat. Photon. 7, 229-233 (2013).

[32] Bash, B. A., Gagatsos, C. N. Datta, A. \& Guha, S. in Proceedings of the 2017 IEEE International Symposium on Information Theory, 3210-3214 (IEEE, 2017).

Acknowledgements: The research is supported by the Australian Research Council (ARC) under the Centre of Excellence for Quantum Computation and Communication Technology (CE110001027). K.L. is supported by the National Natural Science Foundation of China (Grant No. 11674205; 91536222). M.G. acknowledges funding from The National Research Foundation of Singapore (NRF Fellowship Reference No. NRF-NRFF2016-02) and the Singapore Ministry of Education Tier 1 RG190/17. M.G. thanks the Institute of Advanced Study at NTU for funding the travel that catalyzed this work. P.K.L is an ARC Laureate Fellow.

Author contributions: S.A., J.Z., M.G., J.T., and P.K.L. conceived the experiment. S.A. and J.Z. developed the theoretical model. J.Z., K.L., H.J., S.A., and P.K.L. planned and performed the experiment. J.Z. and S.A. analysed the data. J.Z., S.A., H.J., J.T., M.G., and P.K.L. drafted the initial manuscript. All the authors discussed the results and commented on the manuscript. J.Z. and K.L. contribute equally to the work.

Competing interests: The authors declare that they have no competing financial interests.

Additional information:

Correspondence and requests for materials should be addressed to P.K.L and J.Z. 


\section{MATERIALS AND METHODS}

Experimental details. As depicted in Fig. 1, the experiment consists of four parts: a squeezed vacuum source, input preparation, squeezing gate comprised of homodyning and feed-forward, and a homodyne station for characterising the output state. The main light source is a continuous-wave frequency-doubled Nd:YAG laser (Innolight Diablo), producing approximately $300 \mathrm{~mW}$ fundamental wave at $1064 \mathrm{~nm}$ and $400 \mathrm{~mW}$ second harmonic wave at $532 \mathrm{~nm}$. The fundamental beam passes through a mode cleaner cavity with finesse of 760 to further purify its spatial mode and attenuate the highfrequency noise of the laser output. The input coherent states are created by modulating the fundamental beam at $4 \mathrm{MHz}$ sideband frequency using a pair of electrooptical modulators. The squeezed vacuum is prepared in a doubly-resonant bow-tie cavity where below-threshold optical parametric amplification (OPA) takes place using a $10.7 \mathrm{~mm}$ potassium titanyl phosphate (KTP) crystal periodically poled with $9 \mu \mathrm{m}$ period. The front and rear surfaces of the crystal are superpolished and antireflection coated with $\mathrm{R}<0.1 \%$ at $1064 \mathrm{~nm}$ and $\mathrm{R}<0.2 \%$ at $532 \mathrm{~nm}$. Three intracavity mirrors are coated to be highly reflective at both $1064 \mathrm{~nm}$ and $532 \mathrm{~nm}(\mathrm{R}>99.99 \%$ for the two concave mirrors and $\mathrm{R}=(99.85 \pm 0.05) \%$ for the flat mirror), and the input/output coupler has a customised reflection of $(83 \pm 1) \%$ at $1064 \mathrm{~nm}$ and $(73.0 \pm 1.2) \%$ at $532 \mathrm{~nm}$. Up to $11 \mathrm{~dB}$ squeezed vacuum can be generated with a bandwidth of around $36 \mathrm{MHz}$.

Special care was taken in the implementation of all phase locks throughout the experiment. The OPA cavity was locked on co-resonance of both the fundamental beam $(1064 \mathrm{~nm})$ and the pump beam $(532 \mathrm{~nm})$ by means of the Pound-Drever-Hall technique with a $11.25 \mathrm{MHz}$ phase modulation on the pump. The same modulation signal was also utilised to lock the relative phase between the signal beam and the squeezed ancilla, i.e. output of the OPA. The relative phase between the seed and the pump was carefully controlled using a phase modulation at $41.5625 \mathrm{MHz}$ on the seed beam. We use this modulation to ensure that the OPA always operates at the parametric de-amplification, which yields amplitudesqueezed vacuum. The interference between the seed and the local oscillators/auxiliary beam on each homodyne station is controlled similarly with an amplitude modulation $(24.25 \mathrm{MHz})$ and phase modulation $(30 \mathrm{MHz})$ on the signal beam, giving access to the measurement of an arbitrary quadrature angle.

In the experimental investigation of our squeezing gate, we concerned ourselves with five different coherent inputs, each being assigned with a particular target squeezing. By setting $t_{\mathrm{s}}=1$, these input states are characterised by homodyne measurements on amplitude and phase quadratures. The experimental parameters and measured results for the data depicted in Figs. 3-5 are tabulated in the Supplementary Table I.
Filter function. The filter function is used to determine if the squeezing gate operation is successful or not. This is accomplished by picking a random number from a uniform distribution between 0 and 1 and comparing that to $P_{\mathrm{f}}\left(\alpha_{\mathrm{m}}\right)$ (see Eq. 1), where $\alpha_{\mathrm{m}}$ denotes the measurement outcome of the in-loop dual homodyne detection. The operation is heralded as successful when the random number is less than $P_{\mathrm{f}}\left(\alpha_{\mathrm{m}}\right)$. In this case, the measurement outcome is amplified and fed-forward. Otherwise, the operation is considered to have failed and is aborted. The acceptance rate $P_{\mathrm{f}}\left(\alpha_{\mathrm{m}}\right)$ thus determines the likelihood of the acceptance of each outcome, which depends on the amplitude of the input, the filter strength, and the cut-off. For an initial Gaussian distribution of $\alpha_{\mathrm{m}}$, the resultant distribution remains Gaussian provided that the cut-off is sufficiently large, but with its mean and variance both being amplified by $g_{\mathrm{f}}$ [24]. To be concrete, by applying the filter function upon an unnormalised Gaussian ensemble with mean $\alpha_{0}$

$$
e^{-\left|\alpha_{\mathrm{m}}-\alpha_{0}\right|^{2}}
$$

the ensemble evolves as per multiplying Eq. 2 by the acceptance rate $P_{\mathrm{f}}\left(\alpha_{\mathrm{m}}\right)$. The output distribution is essentially a concatenation of two Gaussian distributions joined at the circle $|\alpha|=\alpha_{\mathrm{c}}$. The inputs with $\alpha_{\mathrm{m}}>\alpha_{\mathrm{c}}$ are unaffected, whereas those with $\alpha_{\mathrm{m}}<\alpha_{\mathrm{c}}$ are filtered and become proportional to:

$$
\begin{aligned}
& e^{-\left|\alpha_{\mathrm{m}}-\alpha_{0}\right|^{2}} e^{\left(1-1 / g_{\mathrm{f}}\right)\left(\left|\alpha_{\mathrm{m}}\right|^{2}-\alpha_{\mathrm{c}}^{2}\right)} \\
& =\frac{e^{\left(g_{\mathrm{f}}-1\right)\left|\alpha_{0}\right|^{2}}}{e^{\left(1-1 / g_{\mathrm{f}}\right) \alpha_{\mathrm{c}}^{2}}} \exp \left(-\frac{\left|\alpha_{\mathrm{m}}-g_{\mathrm{f}} \alpha_{0}\right|^{2}}{g_{\mathrm{f}}}\right) \text {. }
\end{aligned}
$$

Note that only the second part (cf. Eq. 3) that undergoes post-selection is desired. Therefore, a sufficient cutoff should be able to embrace this Gaussian as much as possible. To be more explicit, it was proposed in [24] that,

$$
\alpha_{\mathrm{c}}=g_{\mathrm{f}}^{2}\left|\alpha_{\mathrm{m}}\right|+\beta g_{\mathrm{f}} \sigma_{\alpha_{\mathrm{m}}} / \sqrt{2}
$$

Here $\sigma_{\alpha_{\mathrm{m}}}$ is the standard deviation of the input distribution; $\beta$ quantifies how well the cut-off circle embraces the output distribution. Upon restoring the proper normalisation of the output distribution, we obtain the success probability of the filtering operation as

$$
\begin{aligned}
P_{\mathrm{s}} & =\frac{e^{\left(g_{\mathrm{f}}-1\right)\left|\alpha_{0}\right|^{2}}}{\pi e^{\left(1-1 / g_{\mathrm{f}}\right) \alpha_{\mathrm{c}}^{2}}} \iint_{\left|\alpha_{\mathrm{m}}\right|<\alpha_{\mathrm{c}}} \exp \left(-\frac{\left|\alpha_{\mathrm{m}}-g_{\mathrm{f}} \alpha_{0}\right|^{2}}{g_{\mathrm{f}}}\right) \mathrm{d}^{2} \alpha_{\mathrm{m}} \\
& +\frac{1}{\pi} \iint_{\left|\alpha_{\mathrm{m}}\right| \geq \alpha_{\mathrm{c}}} \exp \left(-\left|\alpha_{\mathrm{m}}-\alpha_{0}\right|^{2}\right) \mathrm{d}^{2} \alpha_{\mathrm{m}} .
\end{aligned}
$$

We note that a larger cut-off enables a wider operational range of the filter function, however, at the expense of a decreased success probability. For an input ensemble with a large amplitude, a sufficiently large $\alpha_{\mathrm{c}}$ is required; otherwise, the part of the output distribution beyond $\alpha_{\mathrm{c}}$ 
is subject to distortion [24]. Hence, $\alpha_{\mathrm{c}}$ needs to be carefully tailored according to the input ensemble to ensure a faithful squeezing operation while still maintaining a reasonable probability of success (see Supplementary section $\mathrm{V})$.
Data availability: The data that support the plots within this paper and other findings of this study are available from the corresponding author upon reasonable request. 


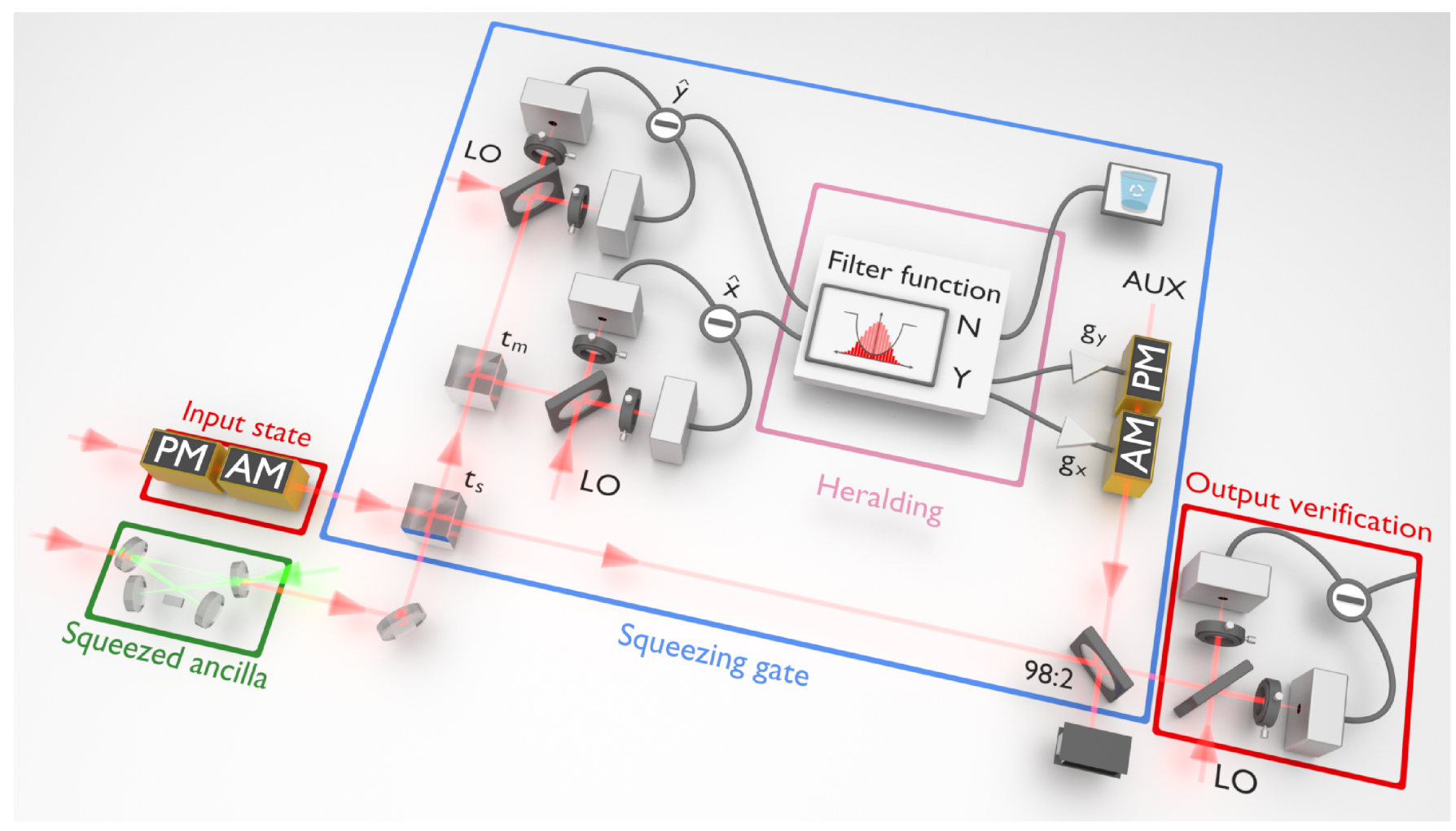

FIG. 1. Experimental layout of the heralded squeezing gate. The gate comprises of three parts. First, the input state and an ancillary squeezed vacuum are prepared. Second, the two states are mixed on a beam splitter with transmissivity of $t_{\mathrm{s}}$. The reflected part of the input state is sent to a dual homodyne measurement where the two conjugate quadratures, amplitude $\hat{X}$ and phase $\hat{Y}$ are measured with a split of $t_{\mathrm{m}}$. This measurement, in conjunction with a heralded filtering function, feedforwarding, and a displacement operation constitute the core of our probabilistic squeezing gate. Lastly, a verification homodyne is employed to characterise the squeezed output. The transmissivities $t_{\mathrm{s}}$ and $t_{\mathrm{m}}$ can be tuned to obtain a trade-off between fidelity and success probability. By setting $t_{\mathrm{m}}=1$ in our experimental demonstration, we obtain a higher success probability compared to the dual homodyne setup without much degradation in the fidelity. AM/PM: electro-optic amplitude/phase modulators; LO: local oscillator; AUX: auxiliary beam. 


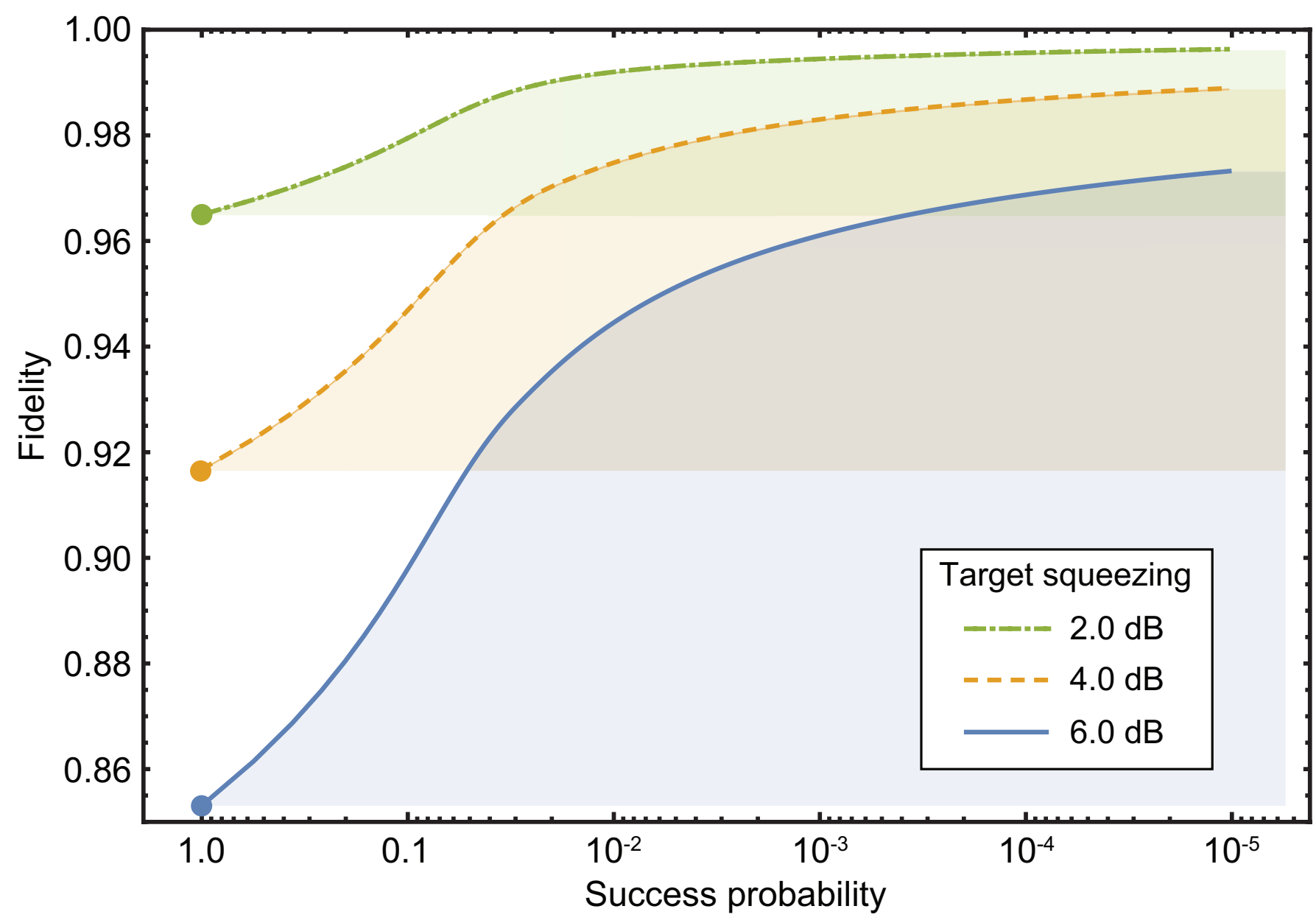

FIG. 2. Fidelity against success probability for various target squeezing. Starting with a pure squeezed ancilla of $6 \mathrm{~dB}$, the fidelity is plotted as a function of success probability for three values of the target squeezing, being $2 \mathrm{~dB}, 4 \mathrm{~dB}$ and $6 \mathrm{~dB}$. For comparison, the fidelity of a conventional deterministic squeezing gate is superimposed (dots). In all cases, a substantial enhancement in fidelity is achieved with the heralded squeezing gate, at the expense of a lower success probability. The cut-off $\alpha_{c}$ is chosen to include more than $98 \%$ of the total statistics to ensure the Gaussianity of the output is preserved (see Supplementary Materials IV). 


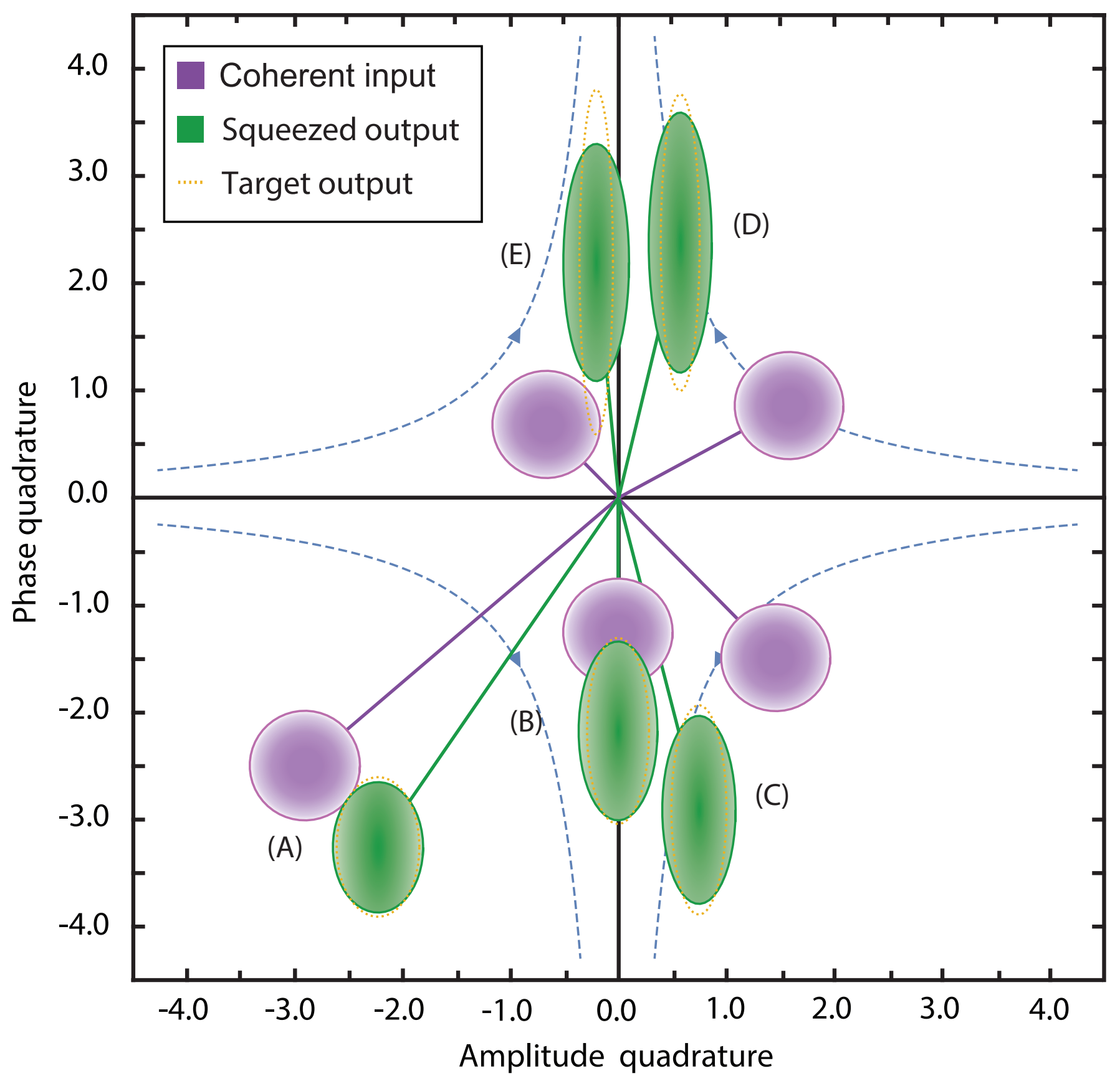

FIG. 3. Phase space diagram for the squeezing gate. To verify the phase invariance of the squeezing operation, five coherent states, labelled $(\mathrm{A})-(\mathrm{E})$, located at different regions of phase space are chosen as inputs. The target squeezings are $2.30 \mathrm{~dB}, 4.81 \mathrm{~dB}, 5.84 \mathrm{~dB}, 8.85 \mathrm{~dB}$, and $10.16 \mathrm{~dB}$, respectively. The corresponding inputs, the experimental and desired squeezed outputs are represented by the noise contours (one standard deviation width) of their Wigner functions. In all circumstances, the squeezing gate behaves consistently irrespective of the input amplitude or phase. 

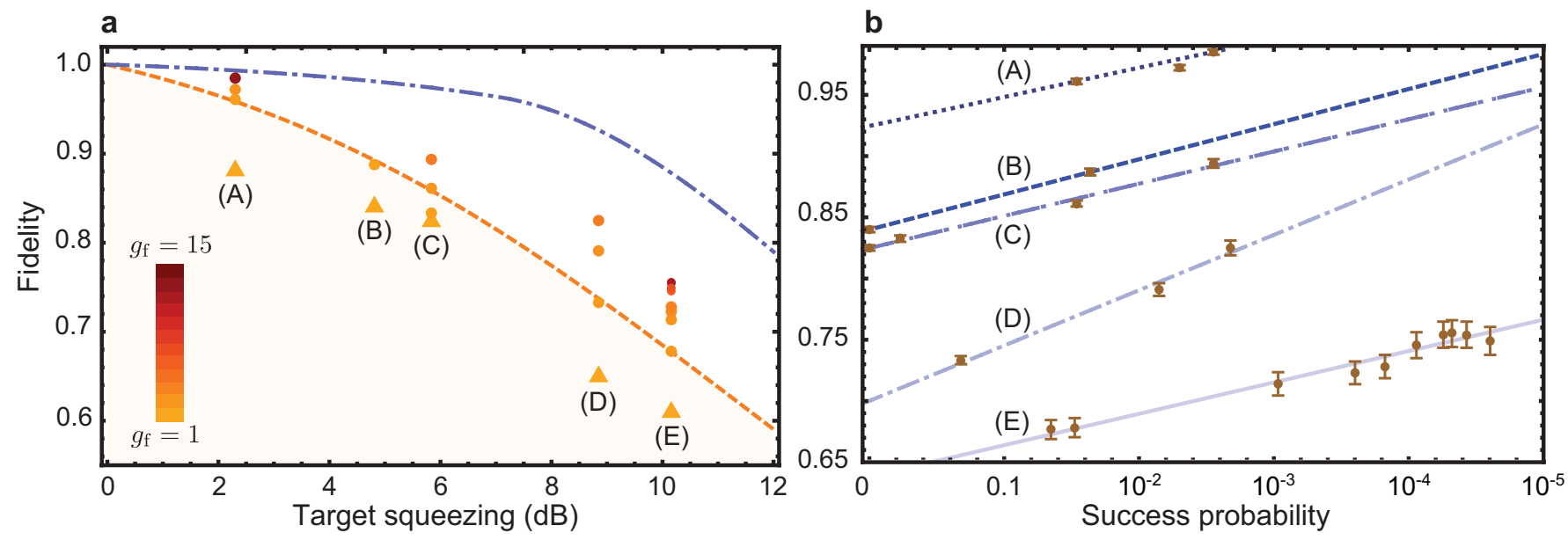

FIG. 4. Improvement in fidelity over conventional techniques for a series of target squeezing for states (A)-(E). a. The optimal fidelities attainable in two scenarios are plotted as performance benchmarks: the presented squeezing gate when a dual homodyne is performed (top blue dash-dotted curve), and a conventional squeezing gate (bottom orange curve). Both lines assume no experimental imperfections, representing the optimal fidelity attainable from our initial squeezed resource. Experimental results with target squeezing between $2.30 \mathrm{~dB}$ and $10.16 \mathrm{~dB}$ are plotted with round markers, showing an increase in fidelity as the filter strength increases (darker gradient colour). The triangle markers denote the fidelity obtained when filter strength is set to one. b. The improvement in fidelity comes at the expense of decreasing success probability. Error bars represent 1 s.d. of the output fidelity (see Supplementary section III).
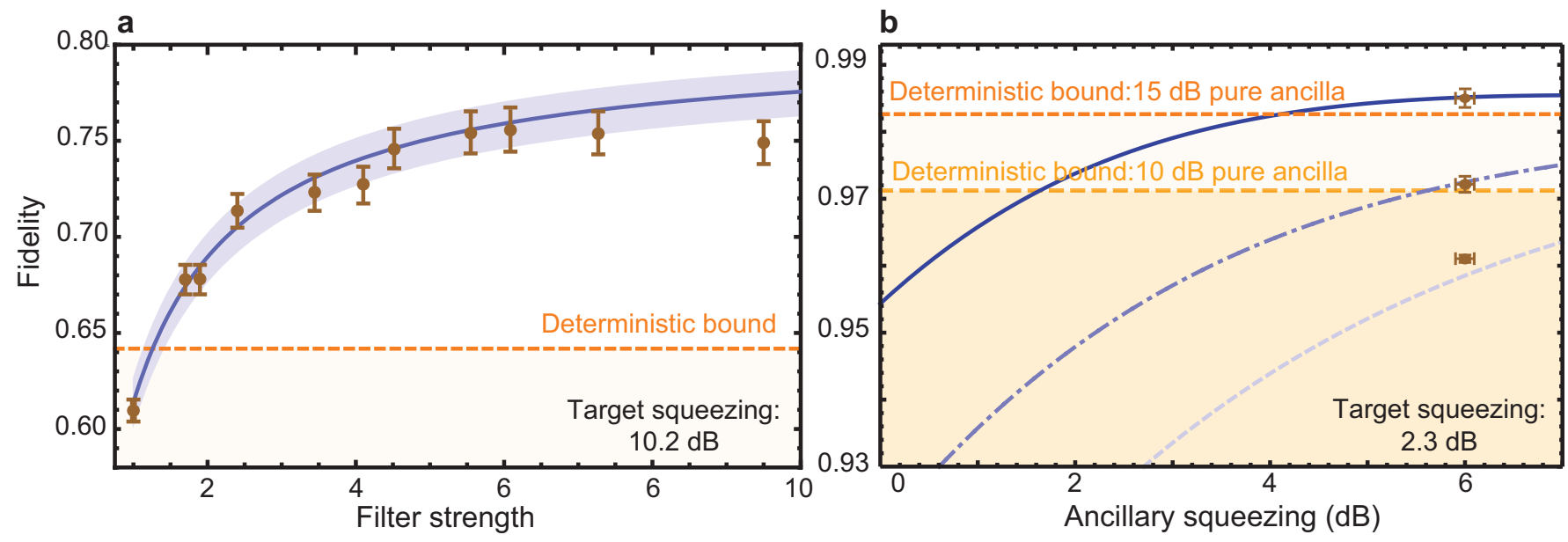

FIG. 5. Fidelity as a function of filter strength and ancillary squeezing. a. The fidelity consistently increases with a higher filter strength, which agrees with the theoretical prediction taking into account of experimental imperfections (blue shaded region). The orange dashed line denotes the limit of fidelity for deterministic protocols assuming pure $6 \mathrm{~dB}$ squeezed ancilla and perfect experimental conditions. Reaching fidelity beyond it would require a larger amount of ancillary squeezing conventionally. b. Filter strength can compensate ancillary squeezing strength. The requirement for a more squeezed ancilla can be circumvented with the presented squeezing gate. The three purple lines, from bottom to top, represent filter strengths of $g_{\mathrm{f}}=1.52,3.38$ and 12.63. For comparison, we present the achievable fidelity for a conventional squeezing gate operating with the currently-best-available squeezing $15 \mathrm{~dB}$ (orange line) and $10 \mathrm{~dB}$ squeezing (yellow line), but sustaining the same in-loop detection efficiency as present in our setup. With $6 \mathrm{~dB}$ of initial squeezing, we observe fidelity surpassing both bounds. Error bars represent 1 s.d. of the output fidelity and the ancillary squeezing. 